The International Glossary on Infertility and Fertility Care, 2017

Zegers-Hochschild, Fernando; Adamson, G. David; Dyer, Silke; Racowsky, Catherine; de Mouzon, Jacques; Sokol, Rebecca; Rienzi, Laura; Sunde, Arne; Schmidt, Lone; Cooke, Ian D.; Leigh Simpson, Joe; van der Poel, Sheryl

Published in:

Human Reproduction

DOI:

10.1093/humrep/dex234

Publication date:

2017

Document version

Publisher's PDF, also known as Version of record

Document license:

CC BY-NC-ND

Citation for published version (APA):

Zegers-Hochschild, F., Adamson, G. D., Dyer, S., Racowsky, C., de Mouzon, J., Sokol, R., Rienzi, L., Sunde, A., Schmidt, L., Cooke, I. D., Leigh Simpson, J., \& van der Poel, S. (2017). The International Glossary on Infertility and Fertility Care, 2017. Human Reproduction, 32(9), 1786-1801. https://doi.org/10.1093/humrep/dex234 


\title{
The International Glossary on Infertility and Fertility Care, $2017^{\dagger+\$}$
}

\author{
Fernando Zegers-Hochschild 1,*, G. David Adamson ${ }^{2}$, Silke Dyer ${ }^{3}$, \\ Catherine Racowsky ${ }^{4}$, Jacques de Mouzon ${ }^{5}$, Rebecca Sokol ${ }^{6}$, \\ Laura Rienzi $^{7}$, Arne Sunde ${ }^{8}$, Lone Schmidt ${ }^{9}$, Ian D. Cooke ${ }^{10}$, \\ Joe Leigh Simpson " ', and Sheryl van der Poel'2
}

\begin{abstract}
'University Diego Portales, Program of Ethics and Public Policies in Human Reproduction; Clinica las Condes, Unit of Reproductive Medicine, Santiago, Chile ${ }^{2}$ ICMART, Palo Alto Medical Foundation Fertility Physicians of Northern California, Palo Alto, CA, USA ${ }^{3}$ Department of Obstetrics \& Gynecology, Groote Schuur Hospital and Faculty of Health Sciences, University of Cape Town, Cape Town, South Africa ${ }^{4}$ Department of Obstetrics, Gynecology and Reproductive Biology, Brigham and Women's Hospital, Harvard Medical School, Boston, MA 021 I5, USA ${ }^{5}$ INSERM, EIM-ESHRE, ICMART, 15 rue Guilleminot, 75014 Paris, France ${ }^{6}$ Department of Medicine and Obstetrics and Gynecology, Keck School of Medicine, University of Southern California, Los Angeles, CA 90007, USA ${ }^{7}$ GENERA Center for Reproductive Medicine, Valle Giulia Clinic, 00197 Rome, Italy ${ }^{8}$ Department of Obstetrics and Gynecology, St. Olav's University Hospital, Trondheim, Norway ${ }^{9}$ Institute of Public Health, University of Copenhagen, Copenhagen, Denmark ${ }^{10}$ Academic Unit of Reproductive and Developmental Medicine, Department of Oncology and Metabolism, University of Sheffield, Sheffield, UK "'March of Dimes Foundation, White Plains, NY, USA ${ }^{12}$ The Population Council, The Rockefeller University, New York, NY 10065, USA
\end{abstract}

\begin{abstract}
'Led by The International Committee for Monitoring Assisted Reproductive Technologies (ICMART) in Partnership with the American Society for Reproductive Medicine (ASRM), European Society of Human Reproduction and Embryology (ESHRE) International Federation of Fertility Societies, (IFFS), March of Dimes (MOD), African Fertility Society (AFS), Groupe Inter-africain d'Etude de Recherche et d'Application sur la Fertilité (GIERAF), Asian Pacific Initiative on Reproduction (ASPIRE), Middle East Fertility Society (MEFS), Red Latinoamericana de Reproducción Asistida (REDLARA), International Federation of Gynecology and Obstetrics (FIGO)
\end{abstract}

*Correspondence address. ICMART c/o International Conference Services (ICS), Suite 300, I20I West Pender Street, Vancouver, BC, Canada V6E2V2. Tel: +I (604) 68I-2I53; E-mail: icmart@icsevents.com

Submitted on May 18, 2017; accepted on June 5, 2017

STUDY QUESTION: Can a consensus and evidence-driven set of terms and definitions be generated to be used globally in order to ensure consistency when reporting on infertility issues and fertility care interventions, as well as to harmonize communication among the medical and scientific communities, policy-makers, and lay public including individuals and couples experiencing fertility problems?

SUMMARY ANSWER: A set of 283 consensus-based and evidence-driven terminologies used in infertility and fertility care has been generated through an inclusive consensus-based process with multiple stakeholders.

WHAT IS KNOWN ALREADY: In 2006 the International Committee for Monitoring Assisted Reproductive Technologies (ICMART) published a first glossary of 53 terms and definitions. In 2009 ICMART together with WHO published a revised version expanded to 87 terms, which defined infertility as a disease of the reproductive system, and increased standardization of fertility treatment terminology. Since 2009, limitations were identified in several areas and enhancements were suggested for the glossary, especially concerning male factor, demography, epidemiology and public health issues.

STUDY DESIGN, SIZE, DURATION: Twenty-five professionals, from all parts of the world and representing their expertise in a variety of sub-specialties, were organized into five working groups: clinical definitions; outcome measurements; embryology laboratory; clinical and laboratory andrology; and epidemiology and public health. Assessment for revisions, as well as expansion on topics not covered by the

‡ESHRE Pages are not externally peer reviewed. The manuscript has been approved by the Executive Committee of ESHRE.

§This article is simultaneously published in Fertility and Sterility.

(C) The Author 2017. Published by Oxford University Press on behalf of the European Society of Human Reproduction and Embryology

This is an Open Access article distributed under the terms of the Creative Commons Attribution-NonCommercial-NoDerivs licence (http://creativecommons.org/licenses/by-nc-nd/4.0/), which permits non-commercial reproduction and distribution of the work, in any medium, provided the original work is not altered or transformed in any way, and that the work is properly cited. For commercial re-use, please contact journals.permissions@oup.com. 
previous glossary, were undertaken. A larger group of independent experts and representatives from collaborating organizations further discussed and assisted in refining all terms and definitions.

PARTICIPANTS/MATERIALS, SETTING, METHODS: Members of the working groups and glossary co-ordinators interacted through electronic mail and face-to-face in international/regional conferences. Two formal meetings were held in Geneva, Switzerland, with a final consensus meeting including independent experts as well as observers and representatives of international/regional scientific and patient organizations.

MAIN RESULTS AND THE ROLE OF CHANCE: A consensus-based and evidence-driven set of 283 terminologies used in infertility and fertility care was generated to harmonize communication among health professionals and scientists as well as the lay public, patients and policy makers. Definitions such as 'fertility care' and 'fertility awareness' together with terminologies used in embryology and andrology have been introduced in the glossary for the first time. Furthermore, the definition of 'infertility' has been expanded in order to cover a wider spectrum of conditions affecting the capacity of individuals and couples to reproduce. The definition of infertility remains as a disease characterized by the failure to establish a clinical pregnancy; however, it also acknowledges that the failure to become pregnant does not always result from a disease, and therefore introduces the concept of an impairment of function which can lead to a disability. Additionally, subfertility is now redundant, being replaced by the term infertility so as to standardize the definition and avoid confusion.

LIMITATIONS, REASONS FOR CAUTION: All stakeholders agreed to the vast majority of terminologies included in this glossary. In cases where disagreements were not resolved, the final decision was reached after a vote, defined before the meeting as consensus if passed with $75 \%$. Over the following months, an external expert group, which included representatives from non-governmental organizations, reviewed and provided final feedback on the glossary.

WIDER IMPLICATIONS OF THE FINDINGS: Some terminologies have different definitions, depending on the area of medicine, for example demographic or clinical as well as geographic differences. These differences were taken into account and this glossary represents a multinational effort to harmonize terminologies that should be used worldwide.

\section{STUDY FUNDING/COMPETING INTERESTS: None.}

TRIAL REGISTRATION NUMBER: N/A.

Key words: glossary / terminologies / infertility / fertility care / ART

\section{Introduction}

Terms and definitions currently used in fertility care, infertility and medically assisted reproduction (MAR) can have different meanings that are dependent upon the setting, their usage in research or clinical interventions, or among diverse populations. This can result in difficulties in standardizing and comparing fertility care interventions and their outcomes, especially on a global level. Furthermore, the way some terms are defined can have an impact on their acceptance and understanding, not only by patients and their health care providers, but also by the public and their policy makers, potentially affecting the manner in which reproductive medicine is practiced and accepted at country level. For example, in countries in which embryo cryopreservation is forbidden by regulation or legislation, the distinction between a zygote and an embryo can have enormous influence on clinical decisionmaking such as the number of oocytes inseminated and/or the number of embryos transferred. Similarly, by defining infertility as a disease of the reproductive system (Zegers-Hochschild et al., 2009a, 2009b), which can lead to disability, equity of access to fertility treatments has been facilitated during debate and decision-making at regional and national levels. These definitions helped to structure the debate concerning key elements of sexual and reproductive rights within the decision by the Inter-American Court of Human Rights that resulted in obliging Costa Rica to re-establish access to fertility care through ART and ensure universal availability (Inter American Court of Human Rights, 20I2). Therefore, an international consensus and evidence-driven set of terms and definitions do benefit communication of a common language among scientific and clinical reproductive medicine communities, but these also can have significant impact on the understanding of the field which drives debate concerning overall access to reproductive health care on a global level.

The first international standardized definitions for reporting ART procedures were published by the International Committee for Monitoring Assisted Reproductive Technologies (ICMART) in 2006 as The ICMART Glossary on ART Terminology (Zegers-Hochschild et al., 2006a, 2006b). This document resulted from an ICMART initiative, presented and documented within the meeting report entitled Medical, Ethical and Social Aspects of Assisted Reproduction and published by the World Health Organization (WHO), in 2002 (Vayena et al., 2002). In 2008, the WHO together with ICMART, the Low-Cost IVF Foundation and The International Federation of Fertility Societies (IFFS) organized an international consensus consultation on 'Assisted Reproductive Technologies: Common Terminology and Management in Low-Resource Settings', again held at the WHO in Geneva, Switzerland. The WHO was responsible for steering and management of the consensus consultation, with expert technical lead provided from ICMART, which resulted in the first revision of the glossary. The main terminology objective during the 2008 large consensus consultation was to expand and improve on an internationally accepted set of definitions that would help standardize and harmonize international data collection in order to monitor the utilization, effectiveness, and safety of ART interventions. In 2009, after review and approval 
through WHO processes, the glossary was published in English simultaneously in the journals Human Reproduction and Fertility and Sterility, and was subsequently translated into Spanish and Portuguese (Red Latinoamericana de Reproducción Asistida, 2010), Dutch (De Neubourg et al., 20I2), Japanese (Ishihara, 20I0) and Russian (Korsak, 20I I).

\section{Working methodology}

In December 2014, the WHO held an expert consultation to review progress on its process of developing guidelines for infertility diagnosis, management and treatment, assessing the available evidence through a detailed systematic review process. During this consultation ICMART, together with $\mathrm{WHO}$ and the other experts, agreed that the 2009 glossary be revised and expanded. This coincided with requests at country level to address topic areas poorly represented in the previous glossary, such as those associated with the male, and also to find clarity in terminologies used in infertility especially in the fields of epidemiology, social science and global public health. This International Glossary on Infertility and Fertility Care, 2017, is a consensus revision to harmonize clinical practice and research, and to inform patients and policy.

The revision process of the glossary since 2006 is described in Fig. I. To generate a revision of the 2009 glossary, the process began with the selection and organization of 25 professionals, from different parts of the world and representing a myriad of organizations covering various sub-specialties, into five working groups according to their expertise in: clinical definitions; outcome measurements; embryology laboratory; clinical and laboratory andrology; and epidemiology and public health. This need for a revision was also in response to a requirement to better define terms associated with outcomes and components that impact fertility treatments which include maternal health risks as well as pregnancy and child outcomes. In 2014, the WHO had participated in the modification of the Consolidated Standards of Reporting Trials (CONSORT), to ensure that the evidence reported from clinical trials, or retrospectively from analysis of clinic practice, would include maternal, neonate and child health outcomes (IMPRINT), (Harbin Consensus Conference Workshop Group, 2014a and Harbin Consensus Conference Workshop Group, $2014 b)$. Thus, this 2017 glossary covers a broad spectrum of terms currently being used in infertility and fertility care.

The responsibility of each working group was to review the existing 2009 definitions and to provide changes or to provide additional terms in order to create the most accurate, current, evidence-based and comprehensive glossary possible for this sector of reproductive health. The lead experts of the glossary revision and the respective lead experts from each of the glossary working groups interacted by electronic mail, at international and regional society meetings, and twice attended consultations held in Geneva, Switzerland.

A consensus expert consultation meeting was held in September 2015. In order to reach agreement, a representative of each of the working groups presented their set of draft terms and definitions to all participants, which included experts in diverse fields as well as representatives from patient groups. When disagreements were not resolved, the final decision was reached after a vote, defined before the meeting as consensus if passed with $75 \%$. The vast majority of terminologies included in this 2017 glossary were agreed upon during the
September, 2015 meeting. Adhering to internationally accepted processes, over the next 9 months an external expert group, which included representatives from non-governmental organizations, reviewed and provided final feedback on the glossary.

In addition to the lead organization (ICMART), professionals representing collaborative organizations included The American Society for Reproductive Medicine (ASRM), ESHRE, the American College of Obstetricians and Gynecologists (ACOG), the International Federation of Gynecology and Obstetrics (FIGO), March of Dimes (MOD), the International Federation of Fertility Societies (IFFS), Red Latinoamericana de Reproducción Asistida (REDLARA), African Fertility Society, (AFS), Groupe Inter-africain d'Etude de Recherche et d'Application sur la Fertilité, (GIERAF), Asian Pacific Initiative on Reproduction (ASPIRE), and Middle East Fertility Society (MEFS). Also, organizations representing infertile persons participated during the consensus meeting, which included RESOLVE in the USA, TRASCENDER in Latin America, Joyce Fertility in Uganda, and Fertility Europe, plus other infertile persons' groups. In addition, representatives from the United Nations Population Fund (UNFPA), Population Council, International Planned Parenthood Federation (IPPF) and the Geneva Foundation for Medical Education and Research (GFMER) also contributed to the consultation. The lead experts of the evidence-synthesis groups who were developing and presenting evidence for $\mathrm{WHO}$ guidelines also participated in the glossary debates. Therefore, 108 international professional experts, including clinicians, basic scientists, epidemiologists and social scientists, along with national and regional representatives of infertile persons, participated in the development of this evidence-base driven glossary.

When developing terms and definitions, special attention was given to possible impacts on ethics and human rights issues as well as recognizing and respecting cultural sensitivities, ethnic minorities and gender equality. Some of these considerations resulted in a consensus modification of the previous definition of 'infertility'. The first modification included broadening the concept of infertility to be an 'impairment of individuals' in their capacity to reproduce, irrespective of whether they have a partner. The revised definition also reinforces the concept of infertility as a disease, which can generate an impairment of function.

As part of a health-related international consensus and evidencedriven process, certain terms and their definitions generated for the glossary needed to be consistent with those existing and currently used by the international fertility community and other international organizations. This was the case when deciding at which gestational age a miscarriage/abortion becomes a stillbirth, as it impacts individual case documentation as well as subsequent calculations of miscarriage and perinatal mortality rates, when reporting on outcomes of medically assisted reproduction (MAR) interventions and specifically of ART either for registries or research.

Many terminologies carry different meanings and thus are repeatedly misused in the literature or social media. Such is the case with the term 'subfertility'. Consensus was reached that this term should be used interchangeably with 'infertility'. It was agreed that 'subfertility' does not define a different or less severe fertility status than infertility, nor is subfertility a condition that exists before infertility is diagnosed. Furthermore, it is crucial to avoid the assumption that a diagnosis of infertility implies sterility, instead the term 'sterility' should be used to define a permanent state of infertility. Thus, a 
"Medical, Ethical and Social Aspects of Assisted Reproduction" meeting.

Geneva, Switzerland, 2001

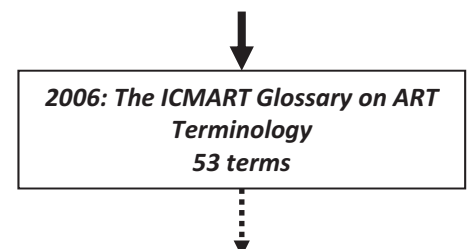

Three working groups:

- Clinical definitions

- Laboratory procedures

- Outcome measures
ICMART/WHO: Consensus consultation on "Assisted Reproductive Technologies: Common Terminology and Management in Low-Resource Settings". Geneva, Switzerland, 2008

$\checkmark$

2009, First revision: The ICMART/WHO Revised Glossary on ART Terminology 87 terms

Five working groups ${ }^{1}$ :

- Clinical definitions

- Outcome measurements

- Embryology laboratory

- Clinical and laboratory andrology

- Epidemiology and public health

-Two international consultations in

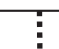

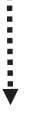

Reassessment of the 2009 glossary:

- Side events at International and Regional society meetings Geneva, Switzerland

-Final international consensus consultation meeting. Geneva, Switzerland. September, 2015

$\downarrow$

2017, Second revision: The International Glossary on Infertility and Fertility Care, 2017. 283 terms
WHO convener.

Non-Governmental

4...... Organizations:

- ICMART

- IFFS

- Low Cost IVF Foundation

\author{
Participating organizations ${ }^{2}$ : \\ ACOG \\ AFS \\ ASPIRE \\ ASRM \\ ESHRE \\ Fertility Europe \\ FIGO \\ GIERAF \\ GFMER \\ IFFS \\ ICMART \\ IPPF \\ Joyce Fertility (Uganda) \\ March of Dimes \\ MEFS \\ Population Council \\ REDLARA \\ RESOLVE (USA) \\ TRASCENDER (Latin America)
}

UNFPA

${ }^{1}$ Names of experts from each working group:

Clinical definitions: Silke Dyer, Osamu Ishihara, Siladitya Bhattacharya, Adam Balen, Herman Tournaye, Paul Devroey, Bart C. Fauser. Outcome measures: Jacques de Mouzon, Ragaa Mansour, Karl-Gösta Nygren, Elizabeth Sullivan, Anja Pinborg, Dolores Lamb.

Embryology laboratory; Clinical and laboratory andrology: Catherine Racowsky, Peter Nagy, Rebecca Sokol, Arne Sunde, Laura Rienzi, Chris Barratt, Lars Bjorndahl, Balaban Basak.

Epidemiology and public health: Ian D Cooke, Lone Schmidt, Cindy Farquhar, John Collins.

Coordinating team: Fernando Zegers-Hochschild, G. David Adamson, Sheryl van der Poel.

${ }^{2}$ Collaborative institutions and their representatives:

ACOG (Robert Rebar), AFS (Oladapo Ashiru, James Olalobo-Lalobo), ASPIRE (Jaideep Malhotra), ASRM (Richard Reindollar, Andrew La Barbera), ESHRE (Kersti Lundin, Roy Farquharson), Fertility Europe (Anna Krawczack), FIGO (Gamal Serour, G. David Adamson), GIERAF (Ernestine Gwet-Bell, Jacques de Mouzon), GFMER (Aldo Campana, Blaise Bourrit), IFFS (Richard Kennedy), ICMART, (G. David Adamson, Silke Dyer), IPPF (Siri Tellier), Joyce Fertility, Uganda (Rita Sembuya), March of Dimes (Joe Leigh Simpson), MEFS (Johnny Awwad, Michel Abou Abdallah), Population Council (Patricia Morris, Sheryl van der Poel), REDLARA (Fernando Zegers-Hochschild), RESOLVE, USA (Barbara Collura), Trascender, Latin America (Carmen Martinez), UNFPA (Luc de Bernis, Michaela Michel-Schuldt).

The experts mentioned above, including additional experts and patient representatives attending the international consensus consultation, represent all global geographic regions: Africa, Europe, Middle East, North and South America, South East Asia and the Western Pacific.

Figure I Review process and methodology of the International Glossary on Infertility and Fertility Care, 20I7. ICMART: International Committee for Monitoring Assisted Reproductive Technologies; WHO: World Health Organization; IFFS: International Federation of Fertility Societies; ACOG: American College of Obstetricians and Gynecologists; ASRM: American Society for Reproductive Medicine; IFFS: International Federation of Fertility Societies; AFS: African Fertility Society; GFMER: Geneva Foundation for Medical Education and Research; GIERAF: Groupe Inter-africain d'Etude de Recherche et d'Application sur la Fertilité; ASPIRE: Asian Pacific Initiative on Reproduction; MEFS: Middle East Fertility Society; REDLARA: Red Latinoamericana de Reproducción Asistida; FIGO: International Federation of Gynecology and Obstetrics; IPPF: International Planned Parenthood Federation; UNFPA: United Nations Population Fund. 
consensus was reached that subfertility is a redundant term, and that the term 'infertility' should be prioritized when providing an assessment or diagnosis of an individual or couple. In a different context, a consensus was also reached to remove from the glossary the term 'conception' and its derivatives such as conceiving or conceived, because these are terms that cannot be described biologically during the process of reproduction. Therefore, instead of using conception, there was consensus that efforts should be made to use scientifically recognized definitions such as fertilization, implantation, pregnancy and live birth. It was also recognized that pregnancies do not 'spontaneously' happen, but rather an act of unprotected sexual intercourse is required; thus, instead of the use of the term 'spontaneous pregnancies' there was consensus that efforts should be made to use the scientifically appropriate terms for pregnancies, either resulting from medical assistance or without assistance.

Consensus terminologies associated with male aspects of infertility were reached that include a clinical perspective as well as terminologies used in the andrology laboratory. Lastly, owing to the lack of standardization in the determination of the burden of infertility, and to better ensure comparability of prevalence data published globally, this revised glossary includes definitions for terms frequently used in epidemiology and public health such as 'voluntary and involuntary childlessness', 'primary and secondary infertility', 'fertility care', 'fecundity' and 'fecundability' among others.

This glossary revision provides the medical and scientific communities, the lay public and policy-makers, as well as individuals and couples experiencing fertility problems or infertility, with a consensus and evidence-driven set of terms and definitions that can be used globally to provide quality care and ensure consistency in registering specific fertility care interventions plus more accurate reporting of their outcomes on national, regional and international registries, and in social media or results from research.

\section{Results}

Led by ICMART in partnership with ASRM, ESHRE, IFFS, MOD, AFS, GIERAF, ASPIRE, MEFS, REDLARA and FIGO, consensus agreement was reached by a global representation of multi-disciplinary experts and patient representatives on 283 terms and definitions which are listed in alphabetical order.

The International Glossary on Infertility and Fertility Care, 2017

\begin{tabular}{|c|c|}
\hline Term & Consensus definition \\
\hline Acrosome & $\begin{array}{l}\text { A membrane-bound structure covering the anterior of the sperm head that contains enzymes necessary } \\
\text { to penetrate the zona pellucida of the oocyte. }\end{array}$ \\
\hline Adenomyosis & $\begin{array}{l}\text { A form of endometriosis marked by the presence of endometrium-like epithelium and stroma outside } \\
\text { the endometrium in the myometrium. }\end{array}$ \\
\hline Adhesions & $\begin{array}{l}\text { Bands of fibrous scar tissue that may bind the abdominal and pelvic organs, including the intestines and } \\
\text { peritoneum, to each other. They can be dense and thick or filmy and thin. }\end{array}$ \\
\hline Age specific fertility rate (ASFR) & $\begin{array}{l}\text { The number of live births per woman in a particular age group in a specific calendar year expressed per } \\
1000 \text { women in that age group. }\end{array}$ \\
\hline Agglutination & Clumping of spermatozoa in the ejaculate. \\
\hline Andrology & The medical practice dealing with the health of the male reproductive system. \\
\hline Aneuploidy & $\begin{array}{l}\text { An abnormal number of chromosomes in a cell. The majority of embryos with aneuploidies are not } \\
\text { compatible with life. }\end{array}$ \\
\hline Anti-sperm antibodies & Antibodies that recognize and bind to antigens on the surface of the spermatozoon. \\
\hline Aspermia & Lack of external ejaculation. \\
\hline Assisted hatching & $\begin{array}{l}\text { An ART procedure in which the zona pellucida of an embryo is either thinned or perforated by chemical, } \\
\text { mechanical or laser methods. }\end{array}$ \\
\hline Assisted reproductive technology (ART) & $\begin{array}{l}\text { All interventions that include the in vitro handling of both human oocytes and sperm or of embryos for the } \\
\text { purpose of reproduction. This includes, but is not limited to, IVF and embryo transfer ET, } \\
\text { intracytoplasmic sperm injection ICSI, embryo biopsy, preimplantation genetic testing PGT, assisted } \\
\text { hatching, gamete intrafallopian transfer GIFT, zygote intrafallopian transfer, gamete and embryo } \\
\text { cryopreservation, semen, oocyte and embryo donation, and gestational carrier cycles. Thus, ART does } \\
\text { not, and ART-only registries do not, include assisted insemination using sperm from either a woman's } \\
\text { partner or a sperm donor. (See broader term, medically assisted reproduction, MAR.) }\end{array}$ \\
\hline Asthenoteratozoospermia & $\begin{array}{l}\text { Reduced percentages of motile and morphologically normal sperm in the ejaculate below the lower } \\
\text { reference limit. When reporting results, the reference criteria should be specified. }\end{array}$ \\
\hline Asthenozoospermia & $\begin{array}{l}\text { Reduced percentage of motile sperm in the ejaculate below the lower reference limit. When reporting } \\
\text { results, the reference criteria should be specified. }\end{array}$ \\
\hline Azoospermia & The absence of spermatozoa in the ejaculate. \\
\hline Binucleation & The presence of two nuclei in a blastomere (cell). \\
\hline
\end{tabular}


Continued

Term

Biochemical pregnancy

Birth (single)

Blastocoele

Blastocyst

Blastomere

Blastomere symmetry

Bleeding after oocyte aspiration

Canceled ART cycle

Childlessness

Chimerism

Cleavage stage embryos

Clinical fertility

Clinical pregnancy

Clinical pregnancy rate

Clinical pregnancy with fetal heart beat

Cohort total fertility rate (CTFR)

Compaction

Complex aneuploidies

Congenital anomalies

Congenital anomaly birth rate

Congenital bilateral absence of the vasa deferentia (CBAVD)

Conventional in vitro insemination

Corona radiata cells

Cross border reproductive care

Cryopreservation

Cryptorchidism

\section{Consensus definition}

A pregnancy diagnosed only by the detection of beta $\mathrm{hCG}$ in serum or urine.

The complete expulsion or extraction from a woman of a fetus after 22 completed weeks of gestational age, irrespective of whether it is a live birth or stillbirth, or, if gestational age is unknown, a birth weight more than 500 grams. A single birth refers to an individual newborn; and a delivery of multiple births, such as a twin delivery, would be registered as two births.

Fluid-filled central region of the blastocyst.

The stage of preimplantation embryo development that occurs around day 5-6 after insemination or ICSI. The blastocyst contains a fluid filled central cavity (blastocoele), an outer layer of cells (trophectoderm) and an inner group of cells (inner cell mass).

A cell in a cleavage stage embryo.

The extent to which all blastomeres are even in size and shape.

Significant bleeding, internal or external, after oocyte aspiration retrieval requiring hospitalization for blood transfusion, surgical intervention, clinical observation or other medical procedure.

An ART cycle in which ovarian stimulation or monitoring has been initiated with the intention to treat, but which did not proceed to follicular aspiration or in the case of a thawed or warmed embryo did not proceed to embryo transfer.

A condition in which a person, voluntarily or involuntarily, is not a legal or societally-recognized parent to a child, or has had all children die.

Presence in a single individual of two or more cell lines, each derived from different individuals.

Embryos beginning with the 2-cell stage and up to, but not including, the morula stage.

The capacity to establish a clinical pregnancy.

A pregnancy diagnosed by ultrasonographic visualization of one or more gestational sacs or definitive clinical signs of pregnancy. In addition to intra-uterine pregnancy, it includes a clinically documented ectopic pregnancy.

The number of clinical pregnancies expressed per 100 initiated cycles, aspiration cycles or embryo transfer cycles. When clinical pregnancy rates are recorded, the denominator (initiated, aspirated or embryo transfer cycles) must be specified.

A pregnancy diagnosed by ultrasonographic or clinical documentation of at least one fetus with a discernible heartbeat.

The observed average number of live born children per woman applied to a birth cohort of women as they age through time. This is obtained from data on women after completing their reproductive years.

The process during which tight junctions form between juxtaposed blastomeres resulting in a solid mass of cells with indistinguishable cell membranes.

Two or more aneuploidies involving different chromosomes in the embryo. When autosomes are involved, this condition is not compatible with human life.

Structural or functional disorders that occur during intra-uterine life and can be identified prenatally, at birth or later in life. Congenital anomalies can be caused by single gene defects, chromosomal disorders, multifactorial inheritance, environmental teratogens and micronutrient deficiencies. The time of identification should be reported.

The number of births exhibiting signs of congenital anomalies per 10,000 births. The time of identification should have been reported.

The absence, at birth, of both duct systems (vas deferentia) that connect the testes to the urethra and may be associated with cystic fibrosis transmembrane conductance regulator (CTFR) gene mutation. Although the testes usually develop and function normally, men present with azoospermia.

The co-incubation of oocytes with sperm in vitro with the goal of resulting in extracorporeal fertilization. The innermost cells of the cumulus oophorus.

The provision of reproductive health services in a different jurisdiction or outside of a recognized national border within which the person or persons legally reside.

The process of slow freezing or vitrification to preserve biological material (e.g. gametes, zygotes, cleavage-stage embryos, blastocysts or gonadal tissue) at extreme low temperature.

Testis not in scrotal position within the neonatal period and, up to but not limited to, I year post birth. If the testis has not descended into the scrotum, this condition can cause primary testicular failure and increased risk of testicular cancer development. 
Continued

Term

Cumulative delivery rate per aspiration/initiated cycle with at least one live birth

\section{Cumulus oophorus}

Cytoplasmic maturation

Cytoplasmic transfer

\section{Decreased spermatogenesis}

Delayed ejaculation

Delayed embryo transfer

Delivery

Delivery rate

Delivery rate after fertility treatment per patient

Delivery with multiple births after fertility treatments

Diandric oocytes

Digynic oocytes

Diminished ovarian reserve

Diploidy/euploidy

Disomy

Donor insemination

Double embryo transfer (DET)

Early neonatal death/mortality

Ectopic pregnancy

Ejaculation

Ejaculation retardata

Ejaculatory duct

Elective embryo transfer

Elective single embryo transfer (eSET)

Embryo

\section{Consensus definition}

The number of deliveries with at least one live birth resulting from one initiated or aspirated ART cycle, including all cycles in which fresh and/or frozen embryos are transferred, until one delivery with a live birth occurs or until all embryos are used, whichever occurs first. The delivery of a singleton, twin, or other multiples is registered as one delivery. In the absence of complete data, the cumulative delivery rate is often estimated.

The multi-layered mass of granulosa cells surrounding the oocyte.

The process during which the oocyte acquires the capacity to support nuclear maturation, fertilization, pronuclei formation, syngamy and subsequent early cleavage divisions until activation of the embryonic genome.

A procedure that can be performed at different stages of an oocyte's development to add to or replace various amounts of cytoplasm from a donor egg.

A histological finding in which spermatogenesis is present with few cells in the seminiferous tubules, resulting in a decreased number or absence of sperm in the ejaculate.

A condition in which it takes a man an extended period of time to reach orgasm and ejaculation.

A procedure in which embryo transfer is not performed within the time frame of the oocyte aspiration cycle but at a later time.

The complete expulsion or extraction from a woman of one or more fetuses, after at least 22 completed weeks of gestational age, irrespective of whether they are live births or stillbirths. A delivery of either a single or multiple newborn is considered as one delivery. If more than one newborn is delivered, it is often recognized as a delivery with multiple births.

The number of deliveries expressed per 100 initiated cycles, aspiration cycles, or embryo transfer cycles. When delivery rates are recorded, the denominator (initiated, aspirated or embryo transfer cycles) must be specified. It includes deliveries that resulted in the birth of one or more live births and/or stillbirths. The delivery of a singleton, twin or other multiple pregnancy is registered as one delivery. If more than one newborn is delivered, it is often recognized as a delivery with multiple births.

The number of deliveries with at least one live birth or stillbirth, expressed per 100 patients, after a specified time and following all treatments.

A single delivery with more than one newborn, following all fertility treatments.

An oocyte with an extra set of haploid chromosomes of paternal origin.

An oocyte with an extra set of haploid chromosomes of maternal origin.

A term generally used to indicate a reduced number and/or reduced quality of oocytes, such that the ability to reproduce is decreased. (See ovarian reserve.)

The condition in which a cell has two haploid sets of chromosomes. Each chromosome in one set is paired with its counterpart in the other set. A diploid embryo has 22 pairs of autosomes and two sex chromosomes, the normal condition.

The normal number of chromosomes characterized by 22 pairs of autosomal chromosomes and one pair of sex chromosomes (XX or $\mathrm{XY}$ ). The chromosome number in human cells is normally 46.

The process of placing laboratory processed sperm or semen from a man into the reproductive tract of a woman who is not his intimate sexual partner, for the purpose of initiating a pregnancy.

The transfer of two embryos in an ART procedure. This may be elective (eDET) when more than two embryos of sufficient quality for transfer are available.

Death of a newborn within 7 days of birth.

A pregnancy outside the uterine cavity, diagnosed by ultrasound, surgical visualization or histopathology. Co-ordinated contractions of the genitourinary tract leading to the ejection of spermatozoa and seminal fluid.

A condition resulting in an inability to ejaculate during vaginal intercourse.

The canal that passes through the prostate just lateral to the verumontanum where the vas deferens and the duct from the seminal vesicle coalesce.

The transfer of one or more embryos, selected from a larger cohort of available embryos.

The transfer of one (a single) embryo selected from a larger cohort of available embryos.

The biological organism resulting from the development of the zygote, until eight completed weeks after fertilization, equivalent to 10 weeks of gestational age. 
Continued

Term

Embryo bank

Embryo donation (for reproduction)

Embryo fragmentation

Embryo recipient cycle

Embryo transfer (ET)

Embryo transfer cycle

Emission (semen)

Endometriosis

Epididymis

Erectile dysfunction

Euploidy

Excessive ovarian response

Expectant fertility management

Extremely low birth weight

Extremely preterm birth

Fecundability

Fecundity

Female infertility

Fertility

Fertility awareness

Fertility care

Fertility preservation

Fertilization

Fetal loss

\section{Consensus definition}

Repository of cryopreserved embryos stored for future use.

An ART cycle, which consists of the transfer of an embryo to the uterus or Fallopian tube of a female recipient, resulting from gametes that did not originate from the female recipient or from her male partner, if present.

The process during which one or more blastomeres shed membrane vesicles containing cytoplasm and occasionally whole chromosomes or chromatin.

An ART cycle in which a woman's uterus is prepared to receive one or more cleavage stage embryos/ blastocysts, resulting from gametes that did not originate from her or from her male partner, if present.

Placement into the uterus of an embryo at any embryonic stage from day I to day 7 after IVF or ICSI. Embryos from day I to day three can also be transferred into the Fallopian tube.

An ART cycle in which one or more fresh or frozen/thawed embryos at cleavage or blastocyst stage are transferred into the uterus or Fallopian tube.

Co-ordinated contractions of the vas deferentia, seminal vesicles, and ejaculatory ducts leading to deposition of semen into the urethral meatus prior to ejaculation.

A disease characterized by the presence of endometrium-like epithelium and stroma outside the endometrium and myometrium. Intrapelvic endometriosis can be located superficially on the peritoneum (peritoneal endometriosis), can extend $5 \mathrm{~mm}$ or more beneath the peritoneum (deep endometriosis) or can be present as an ovarian endometriotic cyst (endometrioma).

A convoluted, highly coiled duct that transports the spermatozoa from the testis via the efferent ducts to the vas deferens.

Inability to have and/or sustain an erection sufficient for intercourse.

The condition in which a cell has chromosomes in an exact multiple of the haploid number; in the human this multiple is normally two. Thus, a normal embryo that is euploid is also diploid.

An exaggerated response to ovarian stimulation characterized by the presence of more follicles than intended. Generally, more than 20 follicles $>12 \mathrm{~mm}$ in size and/or more than 20 oocytes collected following ovarian stimulation are considered excessive, but these numbers are adaptable according to ethnic and other variables.

Management of fertility problems including infertility without any specific active clinical or therapeutic interventions other than fertility information and advice, to improve natural fertility, based upon the probability of becoming pregnant.

Birth weight less than $1000 \mathrm{~g}$.

A birth that takes place after 22 but before 28 completed weeks of gestational age.

The probability of a pregnancy, during a single menstrual cycle in a woman with adequate exposure to sperm and no contraception, culminating in a live birth. In population-based studies, fecundability is frequently measured as the monthly probability.

Clinically defined as the capacity to have a live birth.

Infertility caused primarily by female factors encompassing: ovulatory disturbances; diminished ovarian reserve; anatomical, endocrine, genetic, functional or immunological abnormalities of the reproductive system; chronic illness; and sexual conditions incompatible with coitus.

The capacity to establish a clinical pregnancy.

The understanding of reproduction, fecundity, fecundability, and related individual risk factors (e.g. advanced age, sexual health factors such as sexually transmitted infections, and life style factors such as smoking, obesity) and non-individual risk factors (e.g. environmental and work place factors); including the awareness of societal and cultural factors affecting options to meet reproductive family planning, as well as family building needs.

Interventions that include fertility awareness, support and fertility management with an intention to assist individuals and couples to realize their desires associated with reproduction and/or to build a family.

Various interventions, procedures and technologies, including cryopreservation of gametes, embryos or ovarian and testicular tissue to preserve reproductive capacity.

A sequence of biological processes initiated by entry of a spermatozoon into a mature oocyte followed by formation of the pronuclei.

Death of a fetus. It is referred to as early fetal loss when death takes place between 10 and 22 weeks of gestational age; late fetal loss, when death takes place between 22 and 28 weeks of gestational age; and stillbirth when death takes place after 28 weeks gestational age. 
Continued

Term

Fetus

Freeze-all cycle

Frozen-thawed embryo transfer (FET) cycle

Frozen-thawed oocyte cycle

Full-term birth

Gamete intrafallopian transfer (GIFT)

Germinal vesicle (GV)

Gestational age

Gestational carrier

Gestational sac

Globozoospermia

Haploidy

Hatching

Heterotopic pregnancy

High-order multiple births

High-order multiple gestation

Hydrosalpinx

Hypergonadotropic hypogonadism

Hyperspermia

Hypogonadotropic hypogonadism

Hypospermatogenesis

Hypospermia

latrogenic testicular failure

Immature oocyte

Implantation

Implantation rate

\section{Consensus definition}

The stages of development of an organism from eight completed weeks of fertilization (equivalent to 10 weeks of gestational age) until the end of pregnancy

An ART cycle in which, after oocyte aspiration, all oocytes and/or embryos are cryopreserved and no oocytes and/or embryos are transferred to a woman in that cycle.

An ART procedure in which cycle monitoring is carried out with the intention of transferring to a woman, frozen/thawed or vitrified/warmed embryo(s)/blastocyst(s). Note: A FET cycle is initiated when specific medication is provided or cycle monitoring is started in the female recipient with the intention to transfer an embryo.

An ART procedure in which cycle monitoring is carried out with the intention of fertilizing thawed/ warmed oocytes and performing an embryo transfer.

A birth that takes place between 37 and 42 completed weeks of gestational age.

An ART procedure in which both gametes (oocytes and spermatozoa) are transferred into a Fallopian tube(s).

The nucleus in an oocyte at prophase I.

The age of an embryo or fetus calculated by the best obstetric estimate determined by assessments which may include early ultrasound and the date of the last menstrual period and/or perinatal details. In the case of ART, it is calculated by adding two weeks ( 14 days) to the number of completed weeks since fertilization. Note: For frozen-thawed embryo transfer (FET) cycles, an estimated date of fertilization is computed by subtracting the combined number of days an embryo was in culture pre-cryopreservation and post-thaw/-warm, from the transfer date of the FET cycle.

A woman who carries a pregnancy with an agreement that she will give the offspring to the intended parent(s). Gametes can originate from the intended parent(s) and/or a third party (or parties). This replaces the term 'surrogate.'

A fluid-filled structure associated with early pregnancy, which may be located inside or, in the case of an ectopic pregnancy, outside the uterus.

Describes spermatozoa with a reduced or absent acrosome

The condition in which a cell has one set of each of the 23 single chromosomes. Mature human gametes are haploid, each having 23 single chromosomes.

The process by which an embryo at the blastocyst stage extrudes out of, and ultimately separates from, the zona pellucida.

Concurrent pregnancy involving at least one embryo implanted in the uterine cavity and at least one implanted outside of the uterine cavity.

The complete expulsion or extraction from their mother of three or more fetuses, after 22 completed weeks of gestational age, irrespective of whether they are live births or stillbirths.

A pregnancy with three or more embryos or fetuses.

A distally occluded, dilated, fluid-filled Fallopian tube.

Gonadal failure associated with reduced gametogenesis, reduced gonadal steroid production and elevated gonadotropin production.

High volume of ejaculate above the upper reference limit. When reporting results, the reference criteria should be specified.

Gonadal failure associated with reduced gametogenesis and reduced gonadal steroid production due to reduced gonadotropin production or action.

Histopathologic description of reduced production of spermatozoa in the testes.

Low volume of ejaculate below the lower reference limit. When reporting results, the reference criteria should be specified.

Damage to testicular function after radiation, chemotherapy or hormone treatment; or devascularization as a consequence of hernia surgery.

An oocyte at prophase of meiosis I, (i.e. an oocyte at the germinal vesicle (GV)-stage.)

The attachment and subsequent penetration by a zona-free blastocyst into the endometrium, but when it relates to an ectopic pregnancy, into tissue outside the uterine cavity. This process starts 5 to 7 days after fertilization of the oocyte usually resulting in the formation of a gestation sac.

The number of gestational sacs observed divided by the number of embryos transferred (usually expressed as a percentage, \%). 
Continued

Term

In vitro fertilization (IVF)

In vitro maturation (IVM)

Induced abortion

Induced embryo/fetal reduction

Infertility

Infertility counseling

Initiated medically assisted reproduction cycle (iMAR)

Inner cell mass

Intended parent(s)

Intra-cervical insemination

Intracytoplasmic sperm injection (ICSI)

Intra-uterine insemination

Intra-uterine pregnancy

Laparoscopic ovarian drilling

Large for gestational age

Leukospermia

Leydig cell

Live birth

Live birth delivery rate

Low birth weight

Luteal phase defect

Luteal phase support

Major congenital anomaly

\section{Consensus definition}

A sequence of procedures that involves extracorporeal fertilization of gametes. It includes conventional in vitro insemination and ICSI.

A sequence of laboratory procedures that enable extracorporeal maturation of immature oocytes into fully mature oocytes that are capable of being fertilized with potential to develop into embryos.

Intentional loss of an intrauterine pregnancy, through intervention by medical, surgical or unspecified means. (See induced embryo/fetal reduction.)

An intervention intended to reduce the number of gestational sacs or embryos/fetuses in a multiple gestation.

A disease characterized by the failure to establish a clinical pregnancy after 12 months of regular, unprotected sexual intercourse or due to an impairment of a person's capacity to reproduce either as an individual or with his/her partner. Fertility interventions may be initiated in less than I year based on medical, sexual and reproductive history, age, physical findings and diagnostic testing. Infertility is a disease, which generates disability as an impairment of function.

A professional intervention with the intention to mitigate the physical, emotional and psychosocial consequences of infertility.

A cycle in which the woman receives specific medication for ovarian stimulation or in which cycle monitoring is carried out with the intention to treat, irrespective of whether or not insemination is performed, follicular aspiration is attempted in an ovarian stimulation cycle or whether egg(s) or embryo (s) are thawed or transferred in a frozen embryo transfer (FET) cycle.

A group of cells attached to the polar trophectoderm consisting of embryonic stem cells, which have the potential to develop into cells and tissues in the human body, except the placenta or amniotic membranes.

A couple or person who seek(s) to reproduce with the assistance of a gestational carrier or traditional gestational carrier.

A procedure in which laboratory processed sperm are placed in the cervix to attempt a pregnancy.

A procedure in which a single spermatozoon is injected into the oocyte cytoplasm.

A procedure in which laboratory processed sperm are placed in the uterus to attempt a pregnancy.

A state of reproduction in which an embryo has implanted in the uterus.

A surgical method for inducing ovulation in females with anovulatory or oligo-ovulatory polycystic ovarian syndrome, utilizing either laser or electrosurgery.

A birth weight greater than the 90th centile of the sex-specific birth weight for a given gestational age reference. When reporting outcomes, the reference criteria should be specified. If gestational age is unknown, then the birth weight should be registered.

A high number of white blood cells in semen above the upper reference limit. When reporting results, the reference criteria should be specified.

Type of testicular cell located in the interstitial space between the seminiferous tubules, that secretes testosterone.

The complete expulsion or extraction from a woman of a product of fertilization, after 22 completed weeks of gestational age; which, after such separation, breathes or shows any other evidence of life, such as heart beat, umbilical cord pulsation or definite movement of voluntary muscles, irrespective of whether the umbilical cord has been cut or the placenta is attached. A birth weight of 500 grams or more can be used if gestational age is unknown. Live births refer to the individual newborn; for example, a twin delivery represents two live births.

The number of deliveries that resulted in at least one live birth, expressed per 100 cycle attempts. In the case of ART/MAR interventions, they can be initiated cycles, insemination, aspiration cycles or embryo transfer cycles. When delivery rates are given, the denominator (initiated, inseminated, aspirated or embryo transfer cycles) must be specified.

Birth weight less than $2500 \mathrm{~g}$.

A poorly defined abnormality of the endometrium presumably due to abnormally low progesterone secretion or action on the endometrium.

Hormonal supplementation in the luteal phase, usually progesterone.

A congenital anomaly that requires surgical repair of a defect, is a visually evident or life-threatening structural or functional defect, or causes death. 
Continued

Term

Male infertility

Maternal spindle transfer

Mature oocyte

Maturing oocyte

Medically assisted reproduction (MAR)

Microdissection testicular sperm extraction (MicroTESE)

Micromanipulation in ART

Microsurgical epididymal sperm aspiration/ extraction (MESA/MESE)

Mild ovarian stimulation for IVF

Missed spontaneous abortion/missed miscarriage

Modified natural cycle

Monosomy

Morula

Mosaicism

Multinucleation

Multiple birth

Multiple gestation

Natural cycle ART

Necrozoospermia

Neonatal death/mortality

Neonatal mortality rate

Neonatal period

Non-obstructive azoospermia

Nuclear maturation

Obstructive azoospermia

Oligospermia

Oligozoospermia

\section{Consensus definition}

Infertility caused primarily by male factors encompassing: abnormal semen parameters or function; anatomical, endocrine, genetic, functional or immunological abnormalities of the reproductive system; chronic illness; and sexual conditions incompatible with the ability to deposit semen in the vagina.

Transfer of the maternal spindle (including maternal chromosomes) from a patient's oocyte into a donated oocyte in which the maternal spindle with chromosomes has been removed.

An oocyte at metaphase of meiosis II, exhibiting the first polar body and with the ability to become fertilized.

An oocyte that has progressed from prophase I but has not completed telophase I, thus does not exhibit the first polar body.

Reproduction brought about through various interventions, procedures, surgeries and technologies to treat different forms of fertility impairment and infertility. These include ovulation induction, ovarian stimulation, ovulation triggering, all ART procedures, uterine transplantation and intra-uterine, intracervical and intravaginal insemination with semen of husband/partner or donor.

A surgical procedure using an operating microscope to identify seminiferous tubules that may contain sperm to be extracted for IVF and/or ICSI.

A micro-operative ART procedure performed on sperm, egg or embryo; the most common ART micromanipulation procedures are ICSI, assisted hatching and gamete or embryo biopsy for PGT.

A surgical procedure performed with the assistance of an operating microscope to retrieve sperm from the epididymis of men with obstructive azoospermia. In the absence of optical magnification, any surgical procedure to retrieve sperm from the epididymis should also be registered as MESE.

A protocol in which the ovaries are stimulated with gonadotropins, and/or other pharmacological compounds, with the intention of limiting the number of oocytes following stimulation for IVF.

Spontaneous loss of a clinical pregnancy before 22 completed weeks of gestational age, in which the embryo(s) or fetus(es) is/are nonviable and is/are not spontaneously absorbed or expelled from the uterus.

An ART procedure in which one or more oocytes are collected from the ovaries during a spontaneous menstrual cycle. Pharmacological compounds are administered with the sole purpose of blocking the spontaneous LH surge and/or inducing final oocyte maturation.

The absence of one of the two homologous chromosomes in embryos. Autosomal monosomies in embryos are not compatible with life. Embryos with sex chromosome monosomies are rarely compatible with life.

An embryo formed after completion of compaction, typically 4 days after insemination or ICSI.

A state in which there is more than one karyotypically distinct cell population arising from a single embryo.

The presence of more than one nucleus in a cell.

The complete expulsion or extraction from a woman of more than one fetus, after 22 completed weeks of gestational age, irrespective of whether it is a live birth or stillbirth. Births refer to the individual newborn; for example, a twin delivery represents two births.

A pregnancy with more than one embryo or fetus.

An ART procedure in which one or more oocytes are collected from the ovaries during a menstrual cycle without the use of any pharmacological compound.

The description of an ejaculate in which no live spermatozoa can be found.

Death of a live born baby within 28 days of birth. This can be sub-divided into a) early, if death occurs in the first 7 days after birth; and b) late, if death occurs between 8 and 28 days after birth.

Number of neonatal deaths (up to 28 days) per 1000 live births.

The period which commences at birth and ends at 28 completed days after birth.

Absence of spermatozoa in the ejaculate due to lack of production of mature spermatozoa.

The process during which the oocyte resumes meiosis and progresses from prophase I to metaphase II.

Absence of spermatozoa in the ejaculate due to occlusion of the ductal system.

A term for low semen volume now replaced by hypospermia to avoid confusion with oligozoospermia.

Low concentration of spermatozoa in the ejaculate below the lower reference limit. When reporting results, the reference criteria should be specified. 
Continued

Term

Oocyte

Oocyte aspiration

Oocyte bank

Oocyte donation

Oocyte donation cycle

Oocyte cryopreservation

Oocyte maturation triggering

Oocyte recipient cycle

Oolemma

Ooplasm

Ovarian hyperstimulation syndrome (OHSS)

Ovarian reserve

Ovarian stimulation (OS)

Ovarian tissue cryopreservation

Ovarian torsion

Ovulation

Ovulation induction (OI)

Parthenogenetic activation

Parthenote

Percutaneous epididymal sperm aspiration (PESA)

Perinatal death/mortality

Perinatal mortality rate

Period total fertility rate (PTFR)

Perivitelline space

Pituitary down-regulation

Polar bodies

Polycystic ovary syndrome (PCOS)

\section{Consensus definition}

The female gamete (egg).

Ovarian follicular aspiration performed with the aim of retrieving oocytes.

Repository of cryopreserved oocytes stored for future use.

The use of oocytes from an egg donor for reproductive purposes or research.

An ART cycle in which oocytes are collected from an egg donor for reproductive purposes or research.

The freezing or vitrification of oocytes for future use.

An intervention intended to induce an oocyte in vitro or in vivo to resume meiosis to reach maturity (i.e. to reach metaphase II).

An ART cycle in which a woman receives oocytes from a donor, or her partner if in a same sex relationship, to be used for reproductive purposes.

The cytoplasmic membrane enclosing the oocyte.

The cytoplasm of the oocyte.

An exaggerated systemic response to ovarian stimulation characterized by a wide spectrum of clinical and laboratory manifestations. It may be classified as mild, moderate or severe according to the degree of abdominal distention, ovarian enlargement and respiratory, hemodynamic and metabolic complications.

A term generally used to indicate the number and/or quality of oocytes, reflecting the ability to reproduce. Ovarian reserve can be assessed by any of several means. They include: female age; number of antral follicles on ultrasound; anti-Mullerian hormone levels; follicle stimulating hormone and estradiol levels; clomiphene citrate challenge test; response to gonadotropin stimulation, and oocyte and/or embryo assessment during an ART procedure, based on number, morphology or genetic assessment of the oocytes and/or embryos.

Pharmacological treatment with the intention of inducing the development of ovarian follicles. It can be used for two purposes: I) for timed intercourse or insemination; 2) in ART, to obtain multiple oocytes at follicular aspiration.

The process of slow-freezing or vitrification of tissue surgically excised from the ovary with the intention of preserving reproductive capacity.

Partial or complete rotation of the ovarian vascular pedicle that causes obstruction to ovarian blood flow, potentially leading to necrosis of ovarian tissue.

The natural process of expulsion of a mature egg from its ovarian follicle.

Pharmacological treatment of women with anovulation or oligo-ovulation with the intention of inducing normal ovulatory cycles.

The process by which an oocyte is activated to undergo development in the absence of fertilization.

The product of an oocyte that has undergone activation in the absence of the paternal genome, with (induced) or without (spontaneous) a purposeful intervention.

A surgical procedure in which a needle is introduced percutaneously into the epididymis with the intention of obtaining sperm.

Fetal or neonatal death occurring during late pregnancy (at 22 completed weeks of gestational age and later), during childbirth, or up to seven completed days after birth.

The number of perinatal deaths per 1000 total births (stillbirths plus live births).

The estimated average number of live born children per woman that would be born to a cohort of women throughout their reproductive years, if the fertility rates by age in a given period remained constant at the current age-specific fertility rate.

The space between the cytoplasmic membrane enclosing the oocyte and the innermost layer of the zona pellucida. (This space may contain the first and second polar bodies and extracellular fragments.)

A medical or pharmacological method to prevent the release of gonadotropins (FSH, LH) from the pituitary gland.

The small bodies containing chromosomes segregated from the oocyte by asymmetric division during telophase. The first polar body is extruded at telophase I and normally contains only chromosomes with duplicated chromatids (2c); the second polar body is extruded in response to fertilization or in response to parthenogenetic activation and normally contains chromosomes comprising single chromatids (Ic).

A heterogeneous condition, which requires the presence of two of the following three criteria: (I) Oligoovulation or anovulation; (2) Hyperandrogenism (clinical evidence of hirsutism, acne, alopecia and/or 
Continued

Term

Polycystic ovary (PCO)

Polyploidy

Polyspermy

Poor ovarian responder (POR) in assisted reproductive technology

Poor ovarian response (POR) to ovarian stimulation

Post-implantation embryo

Post-term birth

Posthumous reproduction

Pregnancy

Pregnancy loss

Pregnancy of unknown location (PUL)

Pre-implantation embryo

Preimplantation genetic testing (PGT)

Preimplantation genetic diagnosis (PGD) and screening (PGS)

Premature ejaculation

Premature ovarian insufficiency

Preterm birth

Primary childlessness

Primary female infertility

Primary involuntary childlessness

Primary male infertility

Pronuclei transfer

Pronucleus

Recipient (ART)

\section{Consensus definition}

biochemical hyperandrogenemia); (3) Polycystic ovaries, as assessed by ultrasound scan with more than 24 total antral follicles (2-9 $\mathrm{mm}$ in size) in both ovaries.

An ovary with at least 12 follicles measuring 2-9 $\mathrm{mm}$ in diameter in at least one ovary (Rotterdam criteria). PCO may be present in women with PCOS, but also in women with normal ovulatory function and normal fertility.

The condition in which a cell has more than two haploid sets of chromosomes: e.g. a triploid embryo has three sets of chromosomes and a tetraploid embryo has four sets. Polyploidy in a human embryo is not compatible with life.

The process by which an oocyte is penetrated by more than one spermatozoon.

A woman treated with ovarian stimulation for ART, in which at least two of the following features are present: (I) Advanced maternal age ( $\geq 40$ years); (2) A previous poor ovarian response ( $\leq 3$ oocytes with a conventional stimulation protocol aimed at obtaining more than three oocytes); and, (3) An abnormal ovarian reserve test (i.e. antral follicle count 5-7 follicles or anti-Mullerian hormone $0.5-1.1 \mathrm{ng} / \mathrm{ml}$ (Bologna criteria); or other reference values obtained from a standardized reference population.)

A condition in which fewer than four follicles and/or oocytes are developed/obtained following ovarian stimulation with the intention of obtaining more follicles and oocytes.

An embryo at a stage of development beyond attachment to the endometrium to eight completed weeks after fertilization, which is equivalent to 10 weeks of gestational age.

A live birth or stillbirth that takes place after 42 completed weeks of gestational age.

A process utilizing gametes and/or embryos from a deceased person or persons with the intention of producing offspring.

A state of reproduction beginning with implantation of an embryo in a woman and ending with the complete expulsion and/or extraction of all products of implantation.

The outcome of any pregnancy that does not result in at least one live birth. When reporting pregnancy loss, the estimated gestational age at the end of pregnancy should be recorded.

A pregnancy documented by a positive human chorionic gonadotropin ( $\mathrm{hCG}$ ) test without visualization of pregnancy by ultrasound. This condition exists only after circulating hCG concentration is compatible with ultrasound visualization of a gestational sac.

An embryo at a stage of development beginning with division of the zygote into two cells and ending just prior to implantation into a uterus.

A test performed to analyze the DNA from oocytes (polar bodies) or embryos (cleavage stage or blastocyst) for HLA-typing or for determining genetic abnormalities. These include: PGT for aneuploidies (PGT-A); PGT for monogenic/single gene defects (PGT-M); and PGT for chromosomal structural rearrangements (PGT-SR).

These terms have now been replaced by preimplantation genetic testing PGT. (See term PGT and its definitions.)

A condition in which semen is released sooner than desired.

A condition characterized by hypergonadotropic hypogonadism in women younger than age 40 years (also known as premature or primary ovarian failure). It includes women with premature menopause.

A birth that takes place after 22 weeks and before 37 completed weeks of gestational age.

A condition in which a person has never delivered a live child, or has never been a legal or societallyrecognized parent to a child.

A woman who has never been diagnosed with a clinical pregnancy and meets the criteria of being classified as having infertility.

A condition in a person with a child wish, who has never delivered a live child, or has never been a legal or societally-recognized parent to a child. A major cause of primary involuntary childlessness is infertility.

A man who has never initiated a clinical pregnancy and meets the criteria of being classified as infertile.

Transfer of the pronuclei from a patient's zygote to an enucleated donated zygote.

A round structure in the oocyte surrounded by a membrane containing chromatin. Normally, two pronuclei are seen after fertilization, each containing a haploid set of chromosomes, one set from the oocyte and one from the sperm, before zygote formation.

A person or couple who receives donated eggs, sperm or embryos for the purposes of initiating a pregnancy with the intention of becoming a legally recognized parent. 
Continued

\section{Term}

\section{Recipient ART cycle}

Recurrent spontaneous abortion/miscarriage

Reproductive surgery

Retrograde ejaculation

Salpingectomy

Salpingitis isthmica nodosa (SIN)

Salpingostomy

Secondary female infertility

Secondary involuntary childlessness

Secondary male infertility

Semen analysis

Semen liquefaction

Semen viscosity

Semen volume

Semen/Ejaculate

Seminal plasma

Sertoli cell

Sertoli cell-only syndrome

Severe ovarian hyperstimulation syndrome (OHSS)

Single embryo transfer (SET)

Slow-freezing

Small for gestational age

Sperm bank

Sperm concentration

Sperm density

Sperm isolation

Sperm motility

\section{Consensus definition}

An ART cycle in which a woman receives zygote(s) or embryo(s) from donor(s) or a partner.

The spontaneous loss of two or more clinical pregnancies prior to 22 completed weeks of gestational age.

Surgical procedures performed to diagnose, conserve, correct and/or improve reproductive function in either men or women. Surgery for contraceptive purposes, such as tubal ligation and vasectomy, are also included within this term.

A condition that causes the semen to be forced backward from the ejaculatory ducts into the bladder during ejaculation.

The surgical removal of an entire Fallopian tube.

A nodular thickening of the proximal Fallopian tube (where the tubes join the uterus), which can distort or occlude the tubes and increase the risk of ectopic pregnancy and infertility.

A surgical procedure in which an opening is made in the Fallopian tube either to remove an ectopic pregnancy or open a blocked fluid-filled tube (hydrosalpinx).

A woman unable to establish a clinical pregnancy but who has previously been diagnosed with a clinical pregnancy.

A condition in a person with a child wish, who has previously delivered a live child, or is or has been a legal or societally-recognized parent to a child. A major cause of secondary involuntary childlessness is infertility.

A man who is unable to initiate a clinical pregnancy, but who had previously initiated a clinical pregnancy.

A description of the ejaculate to assess function of the male reproductive tract. Characteristic parameters include volume, $\mathrm{pH}$, concentration, motility, vitality, morphology of spermatozoa and presence of other cells.

The process whereby proteolytic enzymes degrade proteins causing seminal plasma to liquefy.

The description of the relative fluidity of seminal plasma.

The amount of fluid in an ejaculate.

The fluid at ejaculation that contains the cells and secretions originating from the testes and sex accessory glands.

The fluids of the ejaculate.

The non-germinal cell type in the seminiferous tubule that mediates the actions of testosterone and FSH in the testis, provides nutrients and proteins to the developing spermatogenic cells, creates the bloodtestis barrier, and secretes Mullerian-inhibiting hormone.

A condition in which only Sertoli cells line the seminiferous tubules with usually a complete absence of germ cells; also referred to as germ cell aplasia. Spermatogenesis in isolated foci can be observed in rare cases.

A systemic response as a result of ovarian stimulation interventions that is characterized by severe abdominal discomfort and/or other symptoms of ascites, hemoconcentration ( $\mathrm{Hct}>45)$ and/or other serious biochemical abnormalities requiring hospitalization for observation and/or for medical intervention (paracentesis, other).

The transfer of one embryo in an ART procedure. Defined as elective (eSET) when more than one embryo of sufficient quality for transfer is available.

A cryopreservation procedure in which the temperature of the cell(s) is lowered in a step-wise fashion, typically using a computer controlled rate, from physiological (or room) temperature to extreme low temperature.

A birth weight less than the 10th centile for gestational age. When reporting results the reference criteria should be specified. If gestational age is unknown, the birth weight should be registered.

Repository of cryopreserved sperm stored for future use.

The (measure of the) number of spermatozoa in millions per I $\mathrm{ml}$ of semen.

A measure of the mass/volume ratio (specific gravity) for spermatozoa.

A procedure that involves the separation of sperm through centrifugation and resuspension in culture media. It can be used to remove seminal plasma and infectious agents before IUI and ART procedures. This procedure has been shown to be effective in the removal of HIV. It may also be effective in removing other infectious particles but clinical safety and efficacy have to be established for each particular infection. This term is sometimes referred to as 'sperm washing'.

The percentage of moving spermatozoa relative to the total number of spermatozoa. 
Continued

Term

Sperm recipient cycle

Sperm vitality

Spermatogenic arrest

Spermatozoon

Spontaneous abortion/miscarriage

Spontaneous reduction/vanishing sac(s)

Sterility

Stillbirth

Stillbirth rate

Subfertility

Syngamy

Teratozoospermia

Testicular sperm aspiration/extraction (TESA/ TESE)

Thawing

Time to pregnancy (TTP)

Time-lapse imaging

Total delivery rate with at least one live birth

Total fertility rate (TFR)

Total sperm count

Traditional gestational carrier

Trisomy

Trophectoderm

Tubal pathology

Unexplained infertility

Unisomy

\section{Consensus definition}

A MAR cycle in which a woman receives spermatozoa from a person who is not her sexually intimate partner. In the case of ART registry data, a sperm recipient cycle would only include data from cycles using ART procedures.

The percentage of live spermatozoa relative to the total number of spermatozoa.

Failure of germ cells to progress through specific stages of spermatogenesis at onset or during meiosis.

The mature male reproductive cell produced in the testis that has the capacity to fertilize an oocyte. A head carries genetic material, a midpiece produces energy for movement, and a long, thin tail propels the sperm.

The spontaneous loss of an intra-uterine pregnancy prior to 22 completed weeks of gestational age.

The spontaneous disappearance of one or more gestational sacs with or without an embryo or fetus in a multiple pregnancy documented by ultrasound.

A permanent state of infertility.

The death of a fetus prior to the complete expulsion or extraction from its mother after 28 completed weeks of gestational age. The death is determined by the fact that, after such separation, the fetus does not breathe or show any other evidence of life, such as heartbeat, umbilical cord pulsation, or definite movement of voluntary muscles. Note: It includes deaths occurring during labor.

The number of stillbirths per 1000 total births (stillbirths plus live births).

A term that should be used interchangeably with infertility.

The process during which the female and male pronuclei fuse.

A reduced percentage of morphologically normal sperm in the ejaculate below the lower reference limits. When reporting results, the reference criteria should be specified.

A surgical procedure involving one or more testicular biopsies or needle aspirations to obtain sperm for use in IVF and/or ICSI.

The process of raising the temperature of slow-frozen cell(s) from the storage temperature to room/ physiological temperature.

The time taken to establish a pregnancy, measured in months or in numbers of menstrual cycles.

The photographic recording of microscope image sequences at regular intervals in ART, referring to gametes, zygotes, cleavage-stage embryos or blastocysts.

The total number of deliveries with at least one live birth resulting from one initiated or aspirated ART cycle, including all cycles in which fresh and/or frozen embryos are transferred, including more than one delivery from one initiated or aspirated cycle if that occurs, until all embryos are used. Notes: The delivery of a singleton, twin or other multiple pregnancy is registered as one delivery. In the absence of complete data, the total delivery rate is often estimated.

The average number of live births per woman. It may be determined in retrospect, observed data (Cohort Total Fertility Rate, CTFR) or as an estimated average number (Period Total Fertility Rate, PTFR).

The calculated total number of sperm in the ejaculate (semen volume multiplied by the sperm concentration determined from an aliquot of semen).

A woman who donates her oocytes and is the gestational carrier for a pregnancy resulting from fertilization of her oocytes either through an ART procedure or insemination. This replaces the term 'traditional surrogate.'

An abnormal number of chromosome copies in a cell characterized by the presence of three homologous chromosomes rather than the normal two. The majority of human embryos with trisomies are incompatible with life.

Cells forming the outer layer of a blastocyst that have the potential to develop into the placenta and amniotic membranes.

Tubal abnormality resulting in dysfunction of the Fallopian tube, including partial or total obstruction of one or both tubes (proximally, distally or combined), hydrosalpinx and/or peri-tubal and/or peri-ovarian adhesions affecting the normal ovum pick-up function. It usually occurs after pelvic inflammatory disease or pelvic surgery.

Infertility in couples with apparently normal ovarian function, Fallopian tubes, uterus, cervix and pelvis and with adequate coital frequency; and apparently normal testicular function, genito-urinary anatomy and a normal ejaculate. The potential for this diagnosis is dependent upon the methodologies used and/ or those methodologies available.

The condition in a cell resulting from loss of a single chromosome yielding a single copy of that particular chromosome rather than the normal two. The majority of unisomies in human embryos are incompatible with life. 
Continued

Term

Vaginal insemination

Varicocele

Varicocelectomy

Vasectomy

Very low birth weight

Viscosity

Vitrification

Voluntary childlessness

Warming (cells)

Y-chromosome microdeletions

Zona pellucida

Zygote

Zygote intrafallopian transfer (ZIFT)

\section{Consensus definition}

A procedure whereby semen, collected from a non-lubricated condom or similar method, is deposited into the vaginal cavity of a female. An intervention that can be self-administered by a woman attempting pregnancy.

A venous enlargement in the testicular pampiniform plexus.

Procedure to occlude or remove part of the internal spermatic vein in situations in which it has expanded into a varicocele.

Procedure to occlude the vas deferens. It is usually carried out bilaterally in order to secure sterilization.

Birth weight less than $1500 \mathrm{~g}$.

The description of the relative fluidity of the semen.

An ultra-rapid cryopreservation procedure that prevents ice formation within a cell whose aqueous phase is converted to a glass-like solid.

A condition describing a person who does not have or has not had a child wish and does not have any biologically, legally or societally-recognized children.

The process of raising the temperature of a vitrified cell or cells from the storage temperature to room/ physiological temperature.

Missing segments of the genetic material on the $Y$-chromosome that are associated with abnormal spermatogenesis.

The glycoprotein coat surrounding the oocyte.

A single cell resulting from fertilization of a mature oocyte by a spermatozoon and before completion of the first mitotic division.

An ART procedure in which one or more zygotes is transferred into the Fallopian tube.

\section{Authors' roles}

See Fig. I.

\section{Funding}

There was no funding agency for this work.

\section{Conflict of interest}

None declared.

\section{References}

De Neubourg D, van Duijnhoven NTL, Nelen WLDM, D'Hooghe TM. Dutch translation of the ICMART-WHO Revised Glossary on ART Terminology. Gynecol Obstet Invest 20 I ; 74:233-248.

Harbin Consensus Conference Workshop Group. Improving the Reporting of Clinical Trials of Infertility Treatments (IMPRINT): modifying the CONSORT statement. Fertil Steril 20 I4a; 1 02:952-959.

Harbin Consensus Conference Workshop Group. Improving the Reporting of Clinical Trials of Infertility Treatments (IMPRINT): modifying the CONSORT statement. Hum Reprod 20 I4b;29:2075-2082.

Inter-American Court of Human Rights case of Artavia Murillo et al. ('in vitro fertilization') v. Costa Rica judgment of November 28, 2012 (http://www. corteidh.or.cr/docs/casos/articulos/seriec_257_esp.pdf)

Ishihara O ICMART and ICMART/WHO Glossary. Guidebook for Reproductive Medicine. Japan Society for Reproductive Medicine in 2010. ISBN-I0: 430730I056, and ISBN-13: 978-430730I053.
Korsak V. Пересмотренный ИКМАРТ и ВОЗ словарь терминов ВРТ, 2009. Проблемы репродукции 201।;17:7-13.

Red Latinoamericana de Reproducción Asistida. Glosario de Terminología en Tecnicas de Reproducción Asistida (TRA). Versión revisada y preparada por el International Committee for Monitoring Assistede Reproductive Technology (ICMART) y la Organización Mundial de la salud (OMS). JBRA Assist Reprod 20 10; 1 4: 14-23.

Vayena E, Rowe PJ, Griffin PD (eds). Current Practices and Controversies in Assisted Reproduction. Report of a WHO Meeting. Geneva, Switzerland: World Health Organization, 2002, 355-362.

Zegers-Hochschild F, Adamson GD, de Mouzon J, Ishihara O, Mansour R, Nygren K, Sullivan E, van der Poel S, on behalf of ICMART and WHO. The International Committee for Monitoring Assisted Reproductive Technology (ICMART) and the World Health Organization (WHO) revised glossary on ART terminology, 2009. Hum Reprod 2009a;24: 2683-2687.

Zegers-Hochschild F, Adamson GD, de Mouzon J, Ishihara O, Mansour R, Nygren K, Sullivan E, van der Poel S, for ICMART and WHO. International Committee for Monitoring Assisted Reproductive Technology (ICMART) and the World Health Organization (WHO) revised glossary of ART terminology, 2009. Fertil Steril 2009b;92: I520-1524.

Zegers-Hochschild F, Nygren KG, Adamson GD, de Mouzon J, Lancaster P, Mansour R, Sullivan R. The International Committee Monitoring Assisted Reproductive Technologies (ICMART) glossary on ART terminology. Fertil Steril 2006a;86: I 6-19.

Zegers-Hochschild F, Nygren KG, Adamson GD, de Mouzon J, Lancaster P, Mansour R, Sullivan R, on behalf of the International Committee Monitoring Assisted Reproductive Technologies (ICMART). The ICMART glossary on ART terminology. Hum Reprod 2006b;2 I: 1968-1970. 